\title{
Age-Related Hearing Loss: Recent Findings and Emerging Questions
}

\author{
Esperanza Cuadrado ${ }^{1}$, Carolina Sánchez Rodríguez ${ }^{2 *}$ and Ricardo Sanz Fernández ${ }^{2,3}$ \\ 1Department of Clinical Analysis, University Hospital of Getafe, Spain \\ 2Faculty of Biomedical and Health Sciences, European University of Madrid, Spain \\ 3Department of Otorhinolaryngology, University Hospital of Getafe, Spain \\ *Corresponding author: Carolina Sánchez Rodríguez, Faculty of Biomedical and Health Sciences, European University of Madrid, \\ Spain
}

\begin{abstract}
Age-related hearing loss (ARHL) or presbycusis is the most prevalent sensory deficit in the elderly. This progressive hearing impairment leads to social isolation. Currently, our understanding of ARHL is very limited. In this review, we have chosen to focus on recent work that has improved our understanding of the cellular and molecular mechanisms that may cause age-related loss of sensory and neural cells in the cochlea. Our goal here is to give an overview of recent progress towards understanding these phenomena. In addition, we discuss future prospects for advances in our understanding of genetic susceptibility, pathology, and potential therapeutic approaches in ARHL.
\end{abstract}

Keywords: Age-related hearing loss; presbycusis; regenerative therapies; antioxidants; inflammaging; oxidative stress

Abbreviations: ARHL: Age-related hearing loss; WHO: World Health Organization; GPx: Glutathione peroxidase; NAC: N-acetylL-cysteine; VEGF: Vascular Endothelial Grow Factor; BMT: Bone Marrow Transplantation; LGRs: Leucine-rich repeat-containing G-protein coupled Receptors; iPSCs: Induced pluripotent stem cells

\section{Introduction}

Presbycusis, or age-related hearing loss (ARHL), it is a complex and multifactorial disorder, with symmetric and progressive hearing loss over time [1]. According to the World Health Organization (WHO), estimated that in 202570 to $80 \%$ of adults between 65 and 75 years suffer from presbycusis [1], and that the incidence at the beginning of the decade from 2021 to 2030 will be $100 \%$ in adults over 80 years [2]. ARHL is the most prevalent form of hearing loss in humans. It is due to the hearing's deterioriation secondary to the progressive cellular aging of the auditory system, added to external agents that stimulate that damage that is, it is the addition of genetic factors to the cumulative effects of noise, drugs and diseases [3] Hearing loss has an effect on the psychosocial situation of the individual, and, if hearing impairment is not treated, it contributes to social isolation, depression and loss of self-esteem [3]. Given the difficulties of studying presbycusis in human cohorts, researchers have taken to using animal models to help determine the pathogenesis, cellular and molecular mechanisms associated with ARHL. Animal models that, similar to humans, suffer from a progressive decline in hearing, reflected as an increase in auditory thresholds, as age increases [4]. Although not all data obtained from animal models can generalized to humans, animal models are one of the best experimental tools for the evaluation of ARHL.

\section{Molecular Mechanisms of Arhl}

Some theories have been proposed that give a broad overview of the causes at the molecular/cellular level of presbiacusia, focusing mainly on experimental studies in animal models, but the intrinsic mechanisms responsible for hearing loss associated with age have not yet been fully characterized.

\section{Oxidative and nitrosative stress}

Oxidative injury caused by free-radical damage is perhaps the most fundamental cause of age-related pathology in the biological aging of cells. The mitochondrial theory of aging postulates that reactive oxygen species (ROS) generated inside mitochondria 
damage key mitochondrial components, including mtDNA and respiratory chain complex proteins [5]. Therefore, mitochondrial damage and dysfunction are thought to play a central role in ARHL. Age related cochlear hair cell loss is enhanced in mice lacking the antioxidant enzyme Sod1, while mice lacking the antioxidant enzymes Gpx1 or Sod1 show enhanced susceptibility to noiseinduced hearing loss [6]. found that activation of mitochondrial Sirt3 (a member of the sirtuin family) or glutathione reductase, or increased levels of mitochondrial glutathione has great potential for maintaining good hearing by mimicking the anti-aging effects of caloric restriction (CR) in cells of the human inner ear [7]. Our group showed that the levels of ROS/RNS in young rats SD (3 and
6 months) are low, while the levels significantly increase in adult and old rats (12, 18 and 24 months) (Figure 1). We also study the internal protective mechanisms against oxidative stress inside of the cochlea of SD rats. To this end, we measure the total activities of superoxide dismutase (SOD) and glutathione peroxidase (GPx) (antioxidant systems) in the complete homogenized cochlea. At early stages (3months), there is a significant increase in the total SOD and GPx activities in the cochlea, which decreases significantly at later time points (18 and 24 months) (Figure 2). The increase in oxidative and nitrosative stress with age correlates with a significant worsening in ABR and ASSR threshold shifts [8,9].

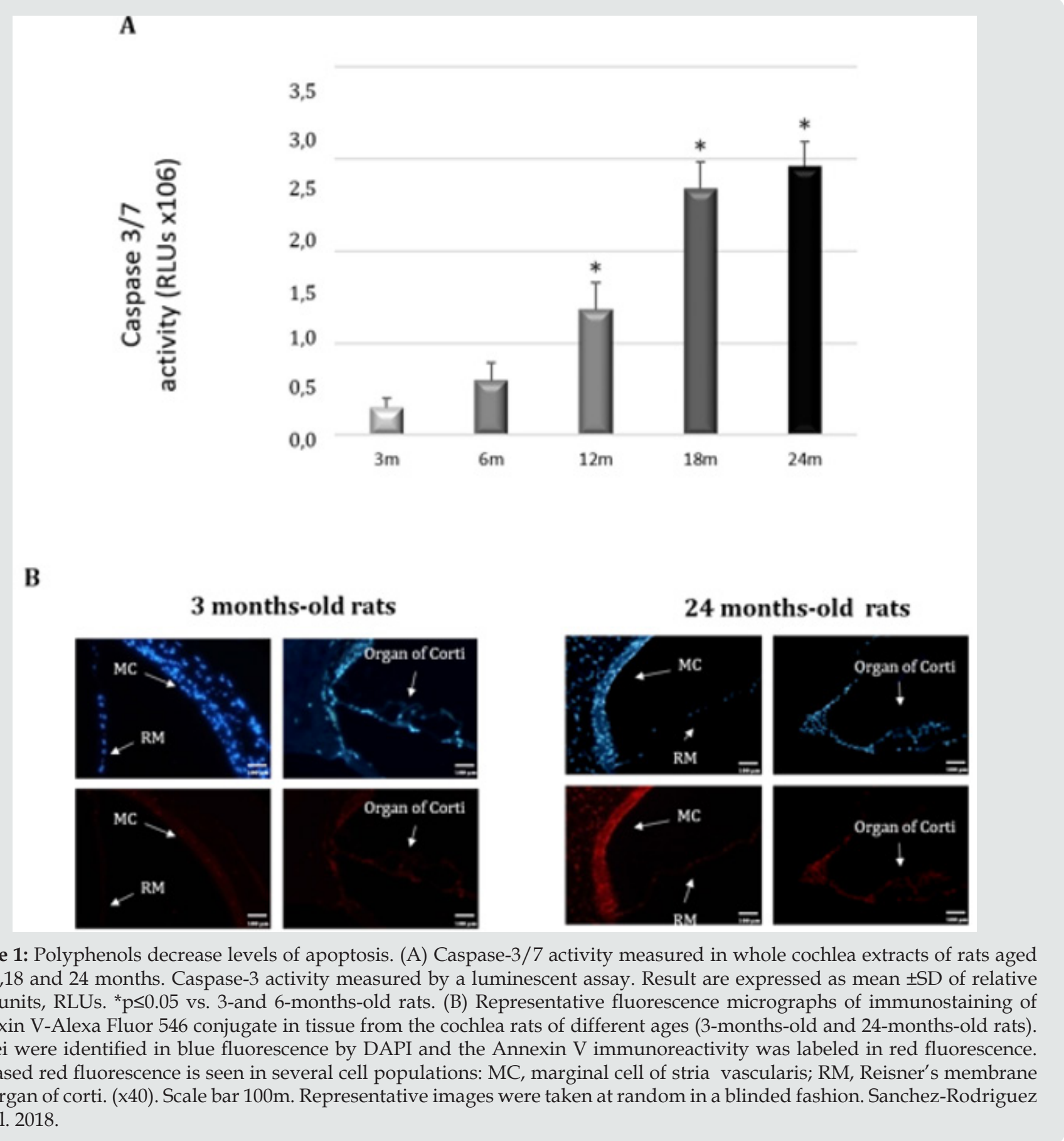

Citation: Esperanza C, Carolina Sánchez R, Ricardo Sanz F. Age-Related Hearing Loss: Recent Findings and Emerging Questions. Sch J Oto 4(1)-2020. SJO. MS.ID.000177. DOI: 10.32474/SJO.2020.04.000177. 


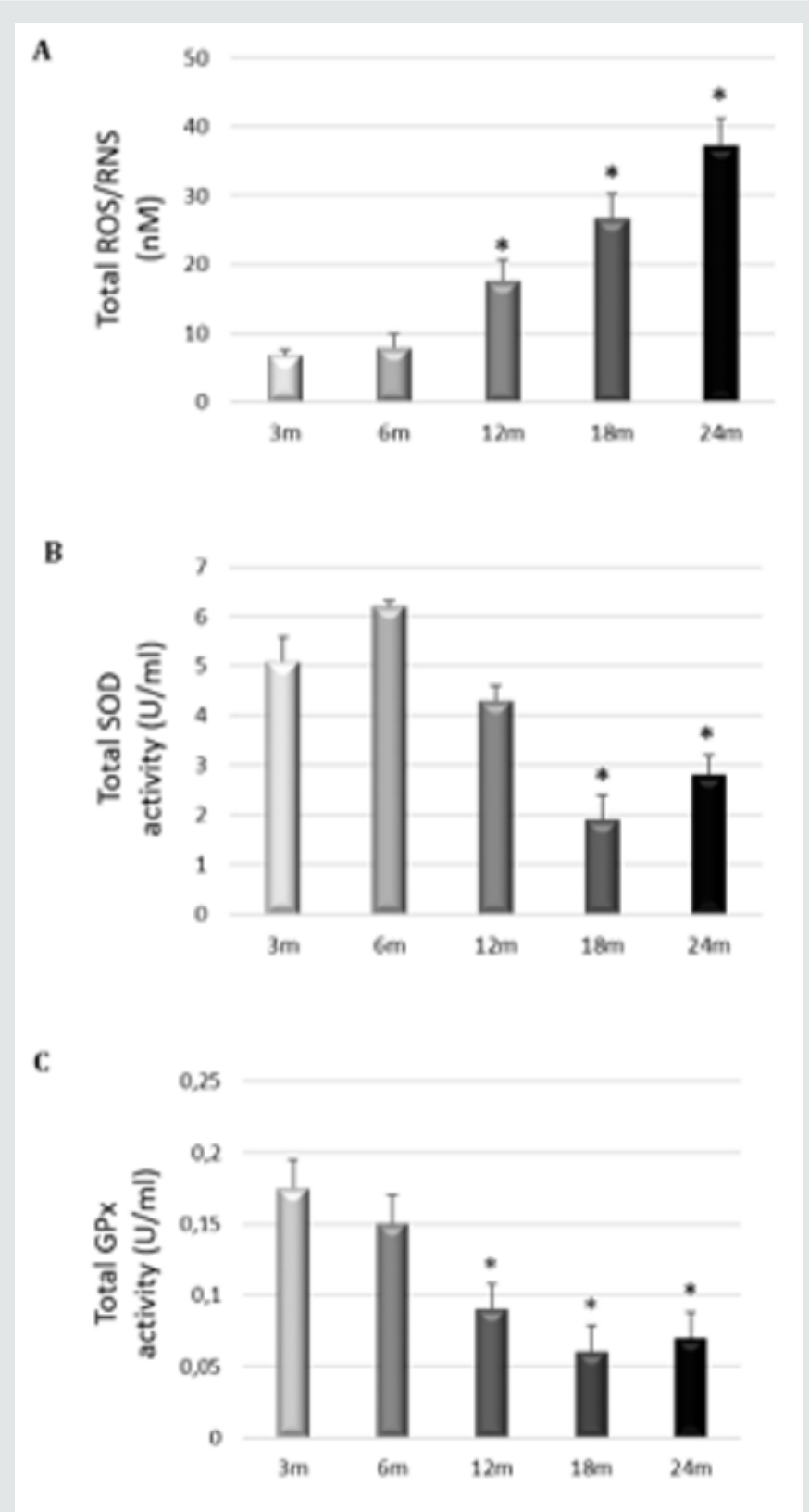

Figure 2: A) Level of ROS/RNS were measured in whole rat cochlear extracts of rats at different. The results are expressed as nM. *p $\leq 0.05$ vs. 3-months-old rats; B) Total SOD and C) GPx activities were measured in whole rat cochlear extracts from rats at different ages. The results are expressed as units/ml. * $\mathrm{p} \leq 0.05$ vs. 3 months-old rats. Sanchez-Rodriguez et al.2016

\section{Cell death pathway}

Many researches have labored to determine if active or passive mechanisms of cell death occur in the cochleae of those with presbycusis. Not surprisingly, researchers have found evidence of both necrosis and programmed cell death in aging cochleae. Multiple lines of evidence suggest that the damage and stress to hair cells and spiral ganglion cells results in programmed cell death. Apoptosis has an important role in pathologies related to aging. For example, mice that overexpress the human X-linked inhibitor of apoptosis (XIAP) protein have less hearing loss than wild-type siblings. Consistently, fewer hair cells died in mice with excess XIAP [10]. While microscopic analysis showed that dying hair cells in the cochleae of older mice typically appeared to be undergoing apoptotic death, there was also evidence of some cells undergoing necrotic death. As might be expected, these authors found expression of molecular markers consistent with caspasedependent and caspase-independent cell death [11]. By contrast, Shen et al. found that either overexpression of the pro-apoptotic protein BCL2 or deletion of the gene encoding that protein affected AHL [12]. However, Someya et al. [13] found that mice with a deletion of the pro-apoptotic gene Bak are resistant to age-related hearing loss and SGN death. In this sense, we previously showed the existence of aging-related induction of caspases in the rat cochlea, evoking the mitochondrion-initiated cell death pathway of 
apoptosis (Figure 3). Activation of the intrinsic mitochondrial cell death pathway usually involves a signal that generates oxidative stress, with the creation of ROS. It is noteworthy that oxidative stress seems to increase with aging and is a major causal factor of cellular damage and enhanced apoptosis in many aging tissues, including the cochlea $[8,9,14]$. Additional work is required to clarify the role of non-apoptotic cell death and to describe the apoptotic pathway functioning in hair cells and SGNs further.
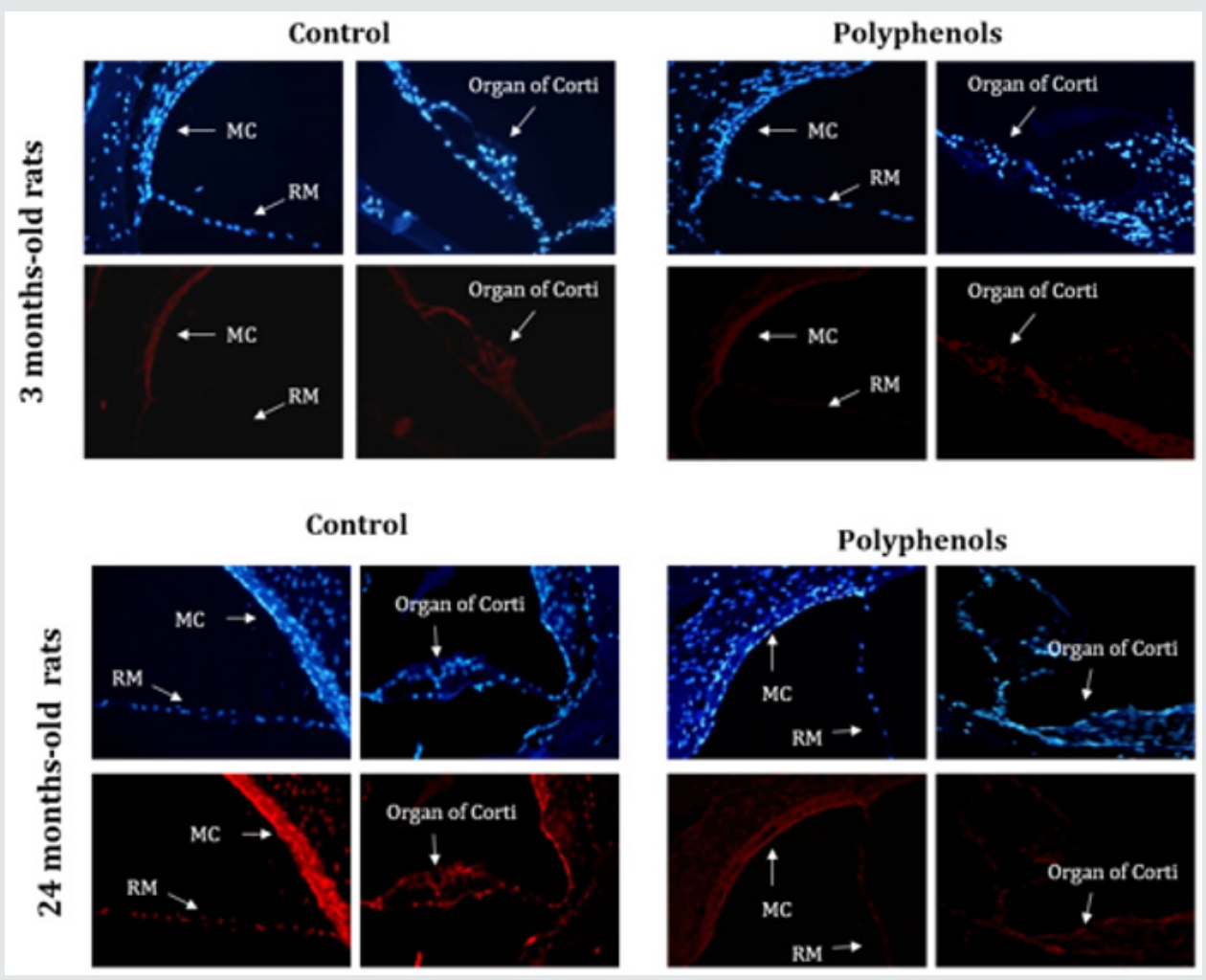

Figure 3: Polyphenols decrease levels of superoxide anions. Young rats,3-months-old and old rats, 24-months-old rats. Superoxide anion detection with fluorescent dihydroethidium probe (red fluorescent) in tissue from the cochlea rats of different ages either untreated or treated with polyphenols. Increased nuclear fluorescence is seen in several cell populations: Mc, marginal cells of stria vascularis; RM, Reisner's membrane and organ of corti magnification x400 . sanchez-Rodriguez C et al.2016.

\section{Inflammaging}

Inflammaging or Chronic inflammation is a ubiquitous characteristic of aging tissue [15]. Chronic inflammation that may begin in a slow, insidious, and even unnoticed manner, which is compatible with the temporal progression of presbycusis. The role of inflammaging in many diverse age-related diseases suggest that inflammaging is an essential component in the physiology of ageing. Several researches have demonstrated that low-grade inflammation processes have critical roles in the causation of agerelated diseases including ARHL. Only a few groups have explored the role of inflammation in the auditory system and most have endorsed a human based cross-sectional analysis approach, so animal model investigations are rare [15]. Verschuur et al. [16] examined relationships between hearing thresholds and key serum biomarkers of low-grade inflammation. They analyzed quantitative relations between the average hearing thresholds of elderly individuals and their IL- 6 and CRP levels, white blood cell counts, as well as neutrophil counts. These data, all precise indications of inflammation, indicated a gradual increase in systemic inflammation as aging persisted as well as a decrease in hearing thresholds. Vershcuur and colleagues concluded that inflammation is a component of the mechanisms underlying Presbycusis. The group of Lowthian et al. [17] is studying the potential therapeutic benefits of low-dose aspirin, a weak anti-inflammatory agent, on ARHL. The aim of the investigation is to determine whether this basic therapy will lessen the progression of the disease in elderly individuals. The results of clinical trial are providing large potential for an inexpensive yet feasible treatment option for preventing or reducing ARHL.

\section{Others pathophysiological theories}

\section{a) Reduction of vascularization in stria vascularis}

In the animal models, signs of the general features of agerelated strial degeneration, including thinning, disorganization, and capillary loss have also been demonstrated. Several mechanisms have been suggested as potentially responsible for the impairment of angiogenesis-associated processes in ageing. First, it has been shown that aged animals display reduced activity of the hypoxiainducible factor-1, resulting in impaired ability to upregulate vascular endothelial grow factor (VEGF) in response to ischemic 
stimuli. Secondly, ageing has been associated with multiple alterations in matrix composition and inflammatory response that are crucial phenomena in physiologic and pathologic angiogenesis and third, senescence influences plasticity and migratory capacities of stem cells and progenitor cells, thus reducing their potential contribution to neo-angiogenic processes. Finally, VEGF induced re-reendothelialization is impaired in aged mice [18,19]. These findings suggest that vascular dysfunction might play a role in ageing-associated hearing

\section{b) Ca2+ signaling pathway}

Aberrant calcium homeostasis has repeatedly been suggested as a contributor to age-related impairment of neuronal function. Hair cells and SGNs have several types of calcium channels, including La T-type voltage-gated calcium channels [20]. Lei et al. [21] showed that wild-type mice treated with T-type calcium channel inhibitors had significant preservation of hearing thresholds and SGNs, as compared to untreated controls. Together, these findings strongly suggest that additional research to study the link between calcium signaling and hearing loss is warranted. Additionally, Mills et al. [22] showed that elderly women using calcium channel blockers were found to have lower hearing thresholds, suggesting that calcium regulation contributes to Presbycusis.

\section{c) Immune pathology}

Aberrant immune cell activity is a contributive factor in the onset and progression of age-related degenerative diseases. This includes disorders such as ARHL. The inner ear mounts an immune response that connects to the systemic immune system and can lead to cochlear degeneration and permanent hearing loss [23]. Iwai et al. [24] have shown that the dysfunctions of the immune system rather than abnormalities in the cochlea cause the development of accelerated sensorineural hearing loss resulting from the deposition of autoantibodies in the cochlea in autoimmune-prone mice. The hearing loss and cochlear pathology can be treated by allogeneic bone marrow transplantation (BMT), replacing the perturbed immune system of the recipient with a new immune system reconstituted by allogeneic donor HSCs.

\section{Therapeutic Perspectives}

Given there is currently an insufficient understanding of the mechanisms involved in ARHL, it is unsurprising that very few clinical trials to prevent or treat ARHL have taken place. The identification of a drug that could prevent or ameliorate further progression of a mild-to-moderate hearing loss is currently a more realistic prospect than a drug or therapy, which can improve an existing hearing loss. Therefore, most studies on new therapies are develop in animal models of ARHL. The increasing rate of age-related hearing loss (ARHL), with its subsequent reduction in quality of life and increase in health care costs, requires new therapeutic strategies to reduce and delay its impact.

\section{Antioxidants}

The basic idea of the antioxidant supplementation interventions for ARHL probably comes from several cross-sectional population- based studies that have showed dietary vitamin intake were significantly associated with the prevalence of age-related hearing loss. The goal of the study of Alvarado et al. [25] was to determine if ARHL could be reduced in a rat model by administering a combination of antioxidant vitamins $\mathrm{A}, \mathrm{C}$, and $\mathrm{E}$ acting as free radical scavengers along with $\mathrm{Mg++}$, a known powerful cochlear vasodilator (ACEMg). These findings indicate that oral ACEMg may provide an effective adjuvant therapeutic intervention for the treatment of ARHL, delaying the progression of hearing impairment associated with age. In Sprague-Dawley animals, our group showed that a mixture of polyphenols with tannic acid, resveratrol, quercitine, rutin, gallic acid, and morin significantly improved ASSR and tone-burst ABR auditory thresholds [26]. In addition, polyphenols reduced oxidative and nitrosative stress, as well as apoptosis in the organ of Corti (Figure 3). These results are related to the significant improvements in the ASSR threshold changes. Some agents, such as N-acetyl-L-cysteine (NAC) [27], CoQ10 [28], acetyl-L-carnitine (ALCAR) [29], and a-lipoic acid [30], have also been studied separately for preventing ARHL. Collectively, although ARHL has been shown to be slowed by supplementation with antioxidants, particularly in laboratory animals, a few studies have investigated the effect of interventions against ARHL in humans. Nevertheless, there is need a long time to observe the preventive effects of antioxidants on ARHL and short-term treatments, such as 1 or 6 months employed in human studies, are not an enough time [31]. The only long-term double-blind, placebo-controlled trial is the Durga's study [32] that was conducted for 3 years and found a minimal but statistically significant reduction in the degree of agerelated hearing loss by folic acid supplement intake. It is clear that defining the type and dosage of antioxidant compounds, timing and duration of the treatment, species, and strains should be account in the future studies.

\section{Gene therapy}

Currently, different molecular therapies are being developed for hearing loss, gene replacement, antisense oligonucleotides, RNA interference and CRISPR-based gene editing.

\section{a) Gene replacement}

Gene replacement is based on identifying and replacing the defective gene with a normal or wild type copy, it's arguably the most 'straight forward' form of gene therapy [33,34]. The first successful inner-ear gene therapy study treated mice homozygous for the targeted deletion of VGLUT3. These mice are born deaf but exogenous replacement of VGLUT3 and its overexpression in inner hair cells (IHCs) mediated by Aden associated virus 1 (AAV1) leads to sustained hearing recovery, partial restoration of ribbon synapse morphology and a startle response [34].

\section{b) Gene suppression-antisense oligonucleotides}

Antisense oligonucleotides (ASOs) are modified nucleic acid sequences that bind to complementary RNA sequences by base pairing. Ponnath et al. [35], injected ASO-29 into homozygous (216AA) and heterozygous (216GA) knock-in mice intraperitoneally at P3 and P10 led to near-normal hearing thresholds in broad band 
noise and 8 and $16 \mathrm{kHz}$ pure tones in 216AA mice treated between P3 and P5. Hearing threshold at $32 \mathrm{kHz}$ could not be rescued, and over the course of 3months there was a gradual decline in thresholds at 8 and $16 \mathrm{kHz}$. The vestibular dysfunction was also rescued. 216AA Mice treated at P10 showed significantly higher thresholds than animals treated at P3-5 but significantly better thresholds than untreated or control-ASO-treated animals in both broad band noise and at $8 \mathrm{kHz}$. Histological assessment of ASO-29treated inner ears demonstrated rescue of outer hair calls (OHCs) and IHCs at P1 bu3t not at P5.

\section{c) Gene suppression-RNA interference}

RNA interference (RNAi) is a biological process in which RNA molecules inhibit gene expression or translation by neutralizing specifically targeted messenger RNA (mRNA) molecules. Its therapeutic use typically focuses on one of two types of small RNA molecule, small interfering RNA (siRNA) or microRNA (miRNA) [36]. While siRNAs and miRNAs share many similarities, their mechanisms of action and clinical applications are different siRNAs tend to be more specific than miRNAs. miRNAs are in general typically regulate the expression of multiple genes [37]. In studies relevant to hearing loss, Shibata et al. [38] designed an artificial miRNA to specifically target the mutation carrying TMC1 allele in the Beethoven mouse, a murine model of human autosomal dominant non-syndromic hearing loss at the DFNA36 locus, and slowed the progressive hearing-loss phenotype in mice treated at P1-2. A follow-up study by Yoshimura et al. [33] treated older animals, and although progression of hearing loss could also be slowed in animals treated at P15 and P30, the results were not as dramatic, and in animals treated at P60, no effect was observed. These findings suggest that, for TMC1-related deafness, the opportunity to intervene using RNAi is temporally defined and, beyond a specific time point, targeted allele suppression has no effect.

\section{d) Gene editing: CRISPR/Cas9}

Targeted genome editing has emerged as a powerful tool for biological research. Although there are three major programmable nucleases, CRISPR (clustered regularly interspaced short palindromic repeats)/Cas9 (CRISPR-associated protein 9)-ZFNs, can identify a target sequence with only $20 \mathrm{bp}$ of guide RNA (gRNA) and also be used for multiplexing by delivering multiple gRNAs to target multiple genes in the same cell at the same time [39]. Gao et al. [40] injected Cas9-gRNA-lipid complexes targeting the mutant TMC1 allele into P1 Beethoven mice and substantially reduced progression of hearing loss. Significant hearing preservation was detected from 8 to $23 \mathrm{kHz}$ with average ABR thresholds $15 \mathrm{~dB}$ lower for treated ears as compared to untreated contralateral ears. This study was the first of its kind to demonstrate the potential of CRISPR/Cas9 for the treatment of autosomal dominant hearing loss related to hair cell dysfunction.

\section{Cell-Based Therapies}

Organ of Corti hair cells are highly specialized mammalian cells; in fact, they are so highly differentiated that they appear to have lost their capacity to divide. Hearing loss in mammals is an irreversible process caused by degeneration of the hair cells of the inner ear. However, some authors believe that some cell populations are able to regenerate after injury, but on lyat very early stages (P0-P3) of development, and not in the adult auditory epithelium [41]. There is great interest in the development of regenerative therapies to treat deafness in the future. However, there are complex factors that need to be considered in application of stem cells therapy, such as stem cell-type choice, signaling pathway regulations, transplantation approaches, internal environment of cochlea and external stimulation.

\section{a) Endogenous stem cell}

In Li et al. [42] found that there were stem cells which have differentiation potential presented in adult mice inner ear, which provides a possibility of application for endogenous stem cell to treat hair cell damage. In Oshima et al. [43]. isolated stem cells from the organ of Corti and vestibular sensory epithelium of newborn mice. These stem cells could differentiate into hair cell-like cells which expressed various hair cell markers and functional ion channels similar to normal hair cells. As the growing of the age, the number of endogenous stem cells also gradually reduced. These problems make the therapeutic potential of cochlear endogenous stem cells only limited to the neonatal mammals with hair cell damage. In the previous study of inner ear development, many genes were considered to participate in regulation of hair cell differentiation process. Their expression products include transcription regulatory factors, growth factors, tyrosine kinase receptor, cell division factordependent kinase inhibitors and Notch receptor, Notch activation molecules, and signaling proteins on the cell membrane surface [44]. A number of studies have demonstrated that the bHLH protein, Atoh1 (also known as Math1) is a key player in embryonic hair cell differentiation [45]. Notch signaling also appears to be activated, impeding the differentiation of some Atoh1 positive cells into hair cells. Recent studies have demonstrated thatthe disruption of Notch signaling results in an increase in the number of hair cells $[46,47]$. Leucine-rich repeat-containing G-protein coupled receptors (LGRs) are also allegedly involved in hair cell regeneration. In particular, Lin et al. [48] suggested that LGR5 positive cells may serve as hair cell progenitors in the mammalian cochlea. Retinoblastoma 1 (RB1) is an endogenous protein that regulating cellular proliferation and differentiation. Downregulation of RB1 was reported to increase the proliferation of hair cells in the cochlea [49]. Findings which have recently emerged have shown that epigenetic mechanisms may also play important roles in controlling stem cell states, and such regulation might also have effects on tissue regeneration [50].

\section{b) Exogenous stem cell}

The other approach is to use exogenous stem cell transplantation to replace damaged or injured cells of cochlea. The low immunogenicity of stem cells, it considered suitable for inner ear application. There are several potential candidates for transplantation to regenerative cells of cochlea like embryonic stem cells (ESCs), Mesenchymal stem cells/Adult stem cells (ASCs) and Induced pluripotent stem cells (iPSCs). ESCs are derived from 
a single fertilized egg. These cells are multipotent so they can differentiate into any cell type. There are many studies about the use of ESCs to regenerate hair cells [51,52], but ESCs can generation of teratomas and to immunological rejection [53]. ESCs introduced directly into the cochlea need to use neurotrophic factors, such as GDNF or BDNF, to promote their survival, although this continues to be quite low [54]. Adult stem cells (ASCs) are undifferentiated cells found throughout the body [55]. Moreover, the use of ASCs in research and therapy is not ethically controversial, because it does not require damage to any embryo, no tumors are created, and ASCs immunoreactivity is very low. The ASCs that have been used for inner ear regeneration are as follows: hematopoietic SCs from bone marrow, mesenchymal SCs and neuronal SCs. One type of ASCs is mesenchymal stem cell (MMSC) that could be used for transplantation, such as bone marrow-derived mesenchymal stem cell, nasal mesenchymal-like stem cell, and adipose-derived mesenchymal stem cell [56-58]. In Cho et al. [59]. transplanted neural differentiated mesenchymal stem cells into the cochlea, but only limited number of regenerated neurons were observed, and mild hearing function was recovered. Some studies have successfully attempted to transfer MSCs to the cochlea to regenerate or replace cochlear fibrocytes. Despite not being sensory epithelium, they play an important role in cochlear physiology and gradually degenerate with age [60].

\section{Delivery systems for ARHL}

Although there is increased understanding of the molecular pathophysiology of ARHL, biologic treatment options are limited due to lack of noninvasive targeted delivery systems. Obstacles of targeted inner ear delivery include anatomic inaccessibility, biotherapeutic instability, and nonspecific delivery. Advances in nanotechnology may provide a solution to these barriers. Nanoparticles can stabilize and carry biomaterials across the round window membrane into the inner ear, and ligand bioconjugation onto nanoparticle surfaces allows for specific targeting. A newer technology, nanohydrogel, may offer noninvasive and sustained biotherapeutic delivery into specific inner ear cells.

\section{a) Nanoparticles}

The delivery of therapeutic molecules with NPs is considered a promising approach for restoring hearing function. NPs can encapsulate various therapeutic agents and deliver them to target cells $[61,62]$. NPs have been created from a variety of materials, generally ranging from tens to several hundred nanometers in diameter [63]. Customization of NP attributes can allow for noninvasive application, drug stabilization, controlled release, and surface modification for specific targeting [64]. Many NP systems have been developed for inner ear drug delivery, including poly (d, l-lactide-co-glycolide acid) (PLGA) nanoparticles (NPs), magnetic NPs, lipid NPs, liposomes, polymersomes, hydroxyapatite NPs, and silica NPs [65]. Certain classes of nanoparticles exhibit additional characteristics that offer unique diagnostic and therapeutic advantages. For example, superparamagnetic iron oxide nanoparticles (SPIONs), gold nanoparticles, and cationic polymer carriers may be used for bioimaging by MRI, confocal laser microscopy, two-photon luminescence, etc. [66-68].
Liposomes containing magnetic resonance imaging (MRI) tracking dye were intratympanic ally injected onto the middleinner ear barriers (oval window and RWM), and the uptake of liposomes in the inner ear was observed using a rat model [69]. Polymerases consisting of amphiphilic block copolymers are vesicular, nanosized spheres that encapsulate an aqueous solution and lead to the delivery of large amounts of biomolecules of interest. Because polymerases are easily modified by chemical conjugation and tagging peptides to target a specific site, promising results, including for OHCs, have been reported [70,71]. While the efficiency of epithelial membrane penetration and cellular uptake should consider in the design of NPs for inner ear delivery, the detection of NPs is also important. When injected, the NPs penetrate the RWM and possibly reach hair cells; thus, the distribution of NPs in the organ of Corti and vestibular tissue indicates the efficacy of the delivery system. In many cases, the direct incorporation of fluorescent dyes is used for optical detection, but other imaging methods e.g. CT, MRI and ultrasound) can also be used if the NPs incorporate proper detection dyes or carbon dots in their innate structure. NP-based drug delivery systems for diseases of the inner ear, a deeper study is needed [70,71].

\section{b) Hydrogels}

An increasing number of approaches have used hydrogels for the delivery of biomolecules to the inner ear by their injection into the middle ear, which causes the biomolecules to diffuse to the inner ear through epithelial membranes. Hydrogels are soft materials networked by physically or chemically crosslinked biopolymers in aqueous solutions [72]. Some hydrogels exhibit a stimuli-responsive property, and thereby undergo an abrupt change in physical properties (i.e. volume, stiffness, degradability and shape) in response to micro-environmental changes, including $\mathrm{pH}$, temperature and enzymes produced by cells they are in contact with [73-75]. PLGA-PEGPLGA hydrogels were used for sustained drug release in guinea pigs through intratympanic injection [76] and this approach was applied in the clinical setting to locally deliver glucocorticoids for hearing recovery in patients with sudden sensorineural hearing loss resistant to systemic treatment [77]. Intratympanic injections of hydrogels of a high viscosity [78] or at a large volume [76] have been found to compromise hearing in guinea pigs.

\section{Conclusion}

While the population of patients with ARHL continues to grow, identification and development of potential protective or therapeutic treatments becomes more and more potentially clinically significant. Compared with the major advances in the understanding of the genes and pathological mechanisms involved in congenital deafness our knowledge of ARHL is still limited. Recent studies in the animal models have effectively revealed numerous cellular and molecular mechanisms that contribute to ARHL. However, it is unclear if these signaling pathways are universally involved in presbycusis. Future studies that rely on more detailed analysis, including cell-type-specific transgenic models, genomic, and proteomic techniques, to ensure the most detailed understanding and effective treatments for presbycusis. 


\section{Acknowledgment}

This research did not receive any specific grant from funding agencies in the public, commercial or not-for-profit sectors.

\section{Conflict of Interest}

The authors declare no conflict of interest.

\section{References}

1. Wattamwar K, Qian ZJ, Otter J, Leskowitz MJ, Caruana FF, et al (2017) Increases in the Rate of Age-Related Hearing Loss in the Older Old. JAMA Otolaryngol Head Neck Surg 143(1): 41-45.

2. Nash SD, Cruickshanks KJ, Klein R, Klein BE, Nieto FJ, et al (2011) The prevalence of hearing impairment and associated risk factors: The Beaver Dam Offspring. Study Arch Otolaryngol Head Neck Surg 137(5): 432-439.

3. Gates GA, Mills JH (2005) Presbycusis. Lancet 366(9491): 1111-1120.

4. Fetoni AR, Picciotti PM, Paludetti G, Troiani D (2011) Pathogenesis of presbycusis in animal models: a review. Exp Gerontol 46(6): 413-425.

5. Tavanai E, Mohammad Hani G (2017) Role of antioxidants in prevention of age-related hearing loss: a review of literature. Eur Arch Otorhinolaryngology 274(4): 1821-1834.

6. Fortunato G, Marciano E, Zarrilli F, Mazzaccara C, Intrieri M, et al. (2004) Paraoxons and superoxide dismutase gene polymorphisms and noiseinduced hearing loss. Clin Chem 50(11): 2012-2018.

7. Han C, Someya S (2013) Maintaining good hearing: calorie restriction Sirt3 and glutathione. Exp Gerontol 48(10): 1091-1095.

8. Sánchez Rodríguez C, Martín Sanz E, Cuadrado E, Granizo JJ, Sanz Fernández R (2016) Protective effect of polyphenols on presbycusis via oxidative/nitrosative stress suppression in rats. Exp Gerontol 83: 31-36.

9. Nevado J, Sanz R, Sánchez Rodríguez C, García Berrocal JR, Martín Sanz E, et al. (2010) Ginkgo biloba extract (EGb761) protects against agingrelated caspase-mediated apoptosis in rat cochlea. Acta Otolaryngol 130(10): 1101-1112

10. Wang J, Menchenton T, Yin S, Yu Z, Bance M, et al. (2010) Over expression of X-linked inhibitor of apoptosis protein slows presbycusis in C57BL/6 J mice. Neurobiol Aging 31(7): 1238-1249.

11. Sha SH, Chen FQ, Schacht J (2009) Activation of cell death pathways in the inner ear of the ageing CBA/J mouse. Hear Res 254(1-2): 92-99.

12. Shen H, Matsui JI, Lei D, Han L, Ohlemiller K, et al. (2010) No dramatic age-related loss of hair cells and spiral ganglion neurons in Bcl-2 overexpression mice or Bax null mice. Mol Neurodegener 5: 28.

13. Someya S, Xu J, Kondo K, Ding D, Salvi RJ, et al. (2009) Age-related hearing loss in C57BL/6 J mice is mediated by Bak-dependent mitochondrial apoptosis. Proc Natl Acad Sci USA 106(46): 19432-19437.

14. Sánchez Rodríguez C, Cuadrado E, Riestra Ayora J, Sanz Fernández R (2018) Polyphenols protect against age-associated apoptosis in female rat cochleae. Biogerontology 19(2): 159-169.

15. Franceschi C, Campisi J (2014) Chronic inflammation (inflammaging) and its potential contribution to age-associated diseases. J Gerontol a Biol Sci Med Sci 69(1): S4-9.

16. Verschuur CA, Dowell A, Syddall HE, Ntani G, Simmonds SJ et al. (2012) Markers of inflammatory status are associated with hearing threshold in older people: findings from the Hertfordshire Ageing Study. Age Ageing 41(1): 92-97.

17. Lowthian JA, Britt CJ, Rance G, Lin FR, Woodset RL, et al. (2016) Slowing the progression of age-related hearing loss: Rationale and study design of the ASPIRIN in HEARING retinal vessels imaging and neurocognition in older generations (ASPREE-hearing) trial. Contemp Clin Trials 46: 6066.
18. Picciotti PM, Fetoni AR, Paludetti G, Wolf FI, Torsello A, et al. (2006) Vascular endothelial growth factor (VEGF) expression in noise-induced hearing loss. Hear Res 214(1-2): 76-83.

19. Fetoni AR, Ferraresi A, Picciotti PM, Gaetani E, Paludetti G, et al. (2009) Noise induced hearing loss and vestibular dysfunction in the guinea pig. Int J Audiol 48(11): 804-810.

20. Lee S, Briklin O, Hiel H, Fuchs P (2007) Calcium-dependent inactivation of calcium channels in cochlear hair cells of the chicken. J Physiol (Lond) 583(3): 909-922.

21. Lei D, Gao X, Perez P, Ohlemiller KK, Chen Ch, et al. (2011) Anti-epileptic drugs delay age-related loss of spiral ganglion neurons via T-type calcium channel. Hearing Research 278(1-2): 106-112.

22. Mills JH, Matthews LJ, Lee FS, Dubno JR, Schulte BA, et al. (1999) Genderspecific effects of drugs on hearing levels of older persons. Ann N Y Acad Sci 884: 381-388.

23. Linton J, Dorshkind K (2004) Age-related changes in lymphocyte development and function. Nat Immunol 5(2): 133-138.

24. Iwai H, Lee S, Inaba M, Baba S, Yamashita T, et al. (2005) Bone marrow transplantation as a strategy for the treatment of autoimmune hearing loss in MRL/Mp-lpr/lpr mice. J Neuroimmunol 168(1-2): 76-82.

25. Alvarado JC, Fuentes Santamaría V, Gabaldón Ul MC, Juiz JM (2018) An Oral Combination of Vitamins A, C, E, and Mg++ Improves Auditory Thresholds in Age-Related Hearing Loss. Front Neurosci 12: 527.

26. Sanz Fernández R, Sánchez Rodriguez C, Granizo JJ, Durio Calero E, Martín Sanz E (2016) Accuracy of auditory steady state and auditory brainstem response to detect the preventive effect to polyphenols on age-related hearing loss in Sprague-Dawley rats. Eur Arch Otorhinolaryngol 273(2): 341-347.

27. Ding D, Jiang H, Chen GD, Longo Guess C, Muthaiah VPK, et al. (2016) $\mathrm{N}$-Acetylcysteine prevents age-related hearing loss and the progressive loss of inner hair cells in c-glutamyl transferase 1 deficient mice. Aging 8(4): 730-750.

28. Salami A, Mora R, Dellepiane M, Manini G, Santomauro V, et al. (2010) Water-soluble coenzyme Q10 formulation (Q-TER) in the treatment of presbycusis. Acta Otolaryngol 130(10): 1154-1162.

29. Bielefeld EC, Coling D, Chen GD, Henderson D (2008) Multiple dosing strategies with acetyl L-carnitine (ALCAR) fail to alter age-related hearing loss in the Fischer 344/NHsd rat. J Negat Results Biomed 7: 4.

30. Peng W, Hu Y, Zhong Y, Chen B, Sun Y, et al. (2010) Protective roles of alpha-lipoic acid in rat model of mitochondrial DNA4834 bp deletion in inner ear. J Huazhong Univ Sci Technol Med Sci 30(4): 514-518.

31. Sable Morita S, Sugiura S, Uchida Y, Tanikawa T, Tokuda H et al. (2018) Relationship between Hearing Impairment and Frailty in Older Patients with Diabetes Mellitus. J Aging Geriatr Med 2(1): 1-4.

32. Durga J, Verhoef P, Anteunis LJ, Schouten E, Kok FJ (2007) Effects of folic acid supplementation on hearing in older adults: a randomized, controlled trial. Ann Intern Med. 146(1): 1-9.

33. Yoshimura H, Shibata SB, Ranum PT, Moteki H, Smith R (2019) Targeted allele suppression prevents progressive hearing loss in the mature murine model of human TMC1 deafness. Mol Ther 27: 681-690.

34. Akil O, Seal RP, Burke K, Wang C, Alemi A, et al. (2012) Restoration of hearing in the VGLUT3 knockout mouse using virally mediated gene therapy. Neuron 75(2): 283-293.

35. Ponnath A, Depreux FF, Jodelka FM, Rigo F, Farris HE, et al. (2018) Rescue of outer hair cells with antisense oligonucleotides in Usher mice is dependent on age of treatment. J Assoc Res Otolaryngol 19(1): 1-16.

36. Adams D, Gonzalez Duarte A, O Riordan WD, Yang Ch, Ueda M, et al. (2018) Patisiran, an RNAi therapeutic, for hereditary transthyretin amyloidosis. N Engl J Med 379: 11-21.

37. Bartel DP (2004) MicroRNAs: genomics, biogenesis, mechanism, and function. Cell 116(2): 281-297. 
38. Shibata SB, Ranum PT, Moteki H, Pan B, Goodwin AT, et al. (2016) RNA interference prevents autosomal-dominant hearing loss. Am J Hum Genet 98(6): 1101-1113.

39. Zou B, Mittal R, Grati M, Lu Z, Shu Y, et al. (2015) The application of genome editing in studying hearing loss. Hear Res 327: 102-108.

40. Gao X, Tao Y, Lamas V, Huang M, Yeh W-H, et al. (2018) Treatment of autosomal dominant hearing loss by in vivo delivery of genome editing agents. Nature 553(7687): 217-221.

41. Kawamoto K, Izumikawa M, Beyer LA, Atkin GM, Raphael Y (2009) Spontaneous hair cell regeneration in the mouse utricle following gentamicin ototoxicity. Hear Res 247(1): 17-26.

42. Li H, Liu H, Heller S (2003) Pluripotent stem cells from the adult mouse inner ear. Nat Med 9(10): 1293-1299.

43. Oshima K, Grimm CM, Corrales CE, Senn P, Martinez Monedero R, et al. (2007) Differential distribution of stem cells in the auditory and vestibular organs of the inner ear. J Assoc Res Otolaryngol 8(1): 18-31.

44. Bermingham McDonogh 0, Reh TA (2011) Regulated reprogramming in the regeneration of sensory receptor cells. Neuron 71(3): 389-405.

45. Jones JM, Montcouquiol M, Dabdoub A, Woods Ch, Kelley MW (2006) Inhibitors of differentiation and DNA binding (Ids) regulate Math1 and hair cell formation during the development of the organ of Corti. J Neurosci 26(2): 550-558

46. Atkinson PJ, Huarcaya Najarro E, Sayyid ZN, Cheng AG (2015) Sensory hair cell development and regeneration: similarities and differences. Development 142(9): 1561-1571.

47. Takebayashi S, Yamamoto N, Yabe D, Fukuda H, Kojima K, et al. (2007) Multiple roles of Notch signaling in cochlear development. Dev Biol 307(1): 165-178

48. Lin J, Zhang X, Wu F, Lin W (2015) Hair cell damage recruited Lgr5expressing cells are hair cell progenitors in neonatal mouse utricle. Front Cell Neurosci 9: 113.

49. Tarang S, Doi SM, Gurumurthy CB, Harms D, Quadros R, Rocha-Sanchez SM (2015) Generation of a Retinoblastoma (Rb)1-inducible dominantnegative (DN) mouse model. Front Cell Neurosci 9: 52

50. Kuo BR, Baldwin EM, Layman WS, Taketo M, Zuo J (2015) In Vivo Cochlear Hair Cell Generation and Survival by Coactivation of $\beta$-Catenin and Atoh1. J Neurosci 35(30): 10786-10798.

51. Yoshikawa M, Ouji Y (2016) Induction of Inner Ear Hair Cells from Mouse Embryonic Stem Cells In Vitro. Methods Mol Biol 1516: 257-267.

52. Longworth Mills E, Koehler KR, Hashino E (2016) Generating Inner Ear Organoids from Mouse Embryonic Stem Cells. Methods Mol Biol 1341: 391-406.

53. Fairchild PJ, Robertson NJ, Minger SL, Waldmann H (2007) Embryonic stem cells: protecting pluripotency from alloreactivity. Curr Opin Immunol 19(5): 596-602.

54. Altschuler RA, OShea KS, Miller JM (2008) Stem cell transplantation for auditory nerve replacement. Hear Res 242(1-2): 110-116.

55. Tarnowski M, Sieron AL (2006) Adult stem cells and their ability to differentiate. Med Sci Monit 12(8): 154-163.

56. Bas E, Van De Water TR, Lumbreras V, Rajguru S, Goss G, et al. (2014) Adult human nasal mesenchymal-like stem cells restore cochlear spiral ganglion neurons after experimental lesion. Stem Cells Dev 23(5): 502514.

57. Boddy SL, Chen W, Romero Guevara R, Kottam L, Bellantuono I, Rivolta MN (2012) Inner ear progenitor cells can be generated in vitro from human bone marrow mesenchymal stem cells. Regen Med 7(6): 757767.

58. Lee JH, Kang WK, Seo JH, Choi MH, Hyun Lee Y, et al. (2012) Neural differentiation of bone marrow-derived mesenchymal stem cells: applicability for inner ear therapy. Korean J Audiol 16(2): 47-53.
59. Cho YB, Cho HH, Jang S, Jeong HS, Park IS (2011) Transplantation of neural differentiated human mesenchymal stem cells into the cochlea of an auditory-neuropathy Guinea pig model. J Korean Med Sci 26(4): 492-498.

60. Kasagi H, Kuhara T, Okada H, Sueyoshi N, Kurihara H (2013) Mesenchymal stem cell transplantation to the mouse cochlea as a treatment for childhood sensorineural hearing loss. Int J Pediatr Otorhinolaryngol 77(6): 936-942.

61. Kim DK (2017) Nanomedicine for inner ear diseases: a review of recent in vivo studies. Biomed Res Int 2017: 3098230

62. Islam MT, Felfel RM, Abou Neel EA, Grant DM, Ahmed I, Zakir Hossain KM (2017) Bioactive calcium phosphate-based glasses and ceramics and their biomedical applications: a review. J Tissue Eng 8: 1-16.

63. Chen G, Zhang X, Yang F, Mu L (2010) Disposition of nanoparticle-based delivery system via inner ear administration. Curr. Drug Metab 11(10): 886-897.

64. Buckiova D, Ranjan S, Newman T, Johnston AH, Sood R et al. (2012) Minimally invasive drug delivery to the cochlea through application of nanoparticles to the round window membrane 7(9): 1339-1354.

65. Justin T, Yajun W, Xiaopei Y, Glynn F, Shepherd RK, Caruso F (2012) Nanoporous peptide particles for encapsulating and releasing neurotrophic factors in an animal model of neurodegeneration. Adv Mater 24(25): 3362-3366.

66. Zou J, Sood R, Ranjan S, Poe d, Ramadanet UA, et al. (2010) Manufacturing and in vivo inner ear visualization of MRI raceable liposome nanoparticles encapsulating gadolinium. J Nanobiotechnology 8: 32.

67. Lai WF and Lin MC (2009) Nucleic acid delivery with chitosan and its derivatives. J Control Release 134(3): 158-168.

68. Lomas H, Johnston APR, Such GK, Zhu Z, Liang K (2011) Polymersomeloaded Capsules for Controlled Release of DNA. Small 7(14): 2109-2119.

69. Christian NA, Milone MC, Ranka SS, Li G, Frail PR, et al. (2007) Tatfunctionalized near-infrared emissive polymersomes for dendritic cell labeling. Bioconjug Chem 18(1): 31-40.

70. Kim YD, Park TE, Singh B, Maharjan S, Choi YJ, et al. (2015) Nanoparticlemediated delivery of siRNA for effective lung cancer therapy. Nanomedicine 10(7): 1165-1188.

71. Yhee JY, Son S, Lee H, Kim K (2015) Nanoparticle-based combination therapy for cancer treatment. Curr Pharm Des 21(22): 3158-3166.

72. Khunmanee S, Jeong Y, Park H (2017) Crosslinking method of hyaluronicbased hydrogel for biomedical applications. J Tissue Eng 8: 1-16.

73. Sood N, Bhardwaj A, Mehta S, Mehta A (2016) Stimuli-responsive hydrogels in drug delivery and tissue engineering. Drug Deliv 23(3): 758-780.

74. Caliari SR and Burdick JA (2016) A practical guide to hydrogels for cell culture. Nat Methods 13(5): 405-414.

75. Khetan S, Guvendiren M, Legant WR, Cohen DM, Chen CS, Burdick JA (2013) Degradationmediated cellular traction directs stem cell fate in covalently crosslinked three-dimensional hydrogels. Nat Mater 12(5): 458-465.

76. Feng L, Ward JA, Li SK, Tolia G, Hao J, Choo DI (2014) Assessment of PLGA-PEG-PLGA copolymer hydrogel for sustained drug delivery in the ear. Curr Drug Deliv 11(2): 279-286.

77. Hütten M, Dhanasingh A, Hessler R, Stöver T, Esser KH (2014) In vitro and in vivo evaluation of a hydrogel reservoir as a continuous drug delivery system for inner ear treatment. PLoS ONE 9(8): e104564.

78. Engmer Berglin C, Videhult Pierre P, Ekborn A, Bramer T, Edsman K, et al. (2015) Local treatment of the inner ear: a study of three different polymers aimed for middle ear administration. Acta Otolaryngol 135(10): 985-994. 
(c) (i) This work is licensed under Creative

To Submit Your Article Click Here: Submit Article

DOI: $10.32474 /$ SJO.2020.04.000177

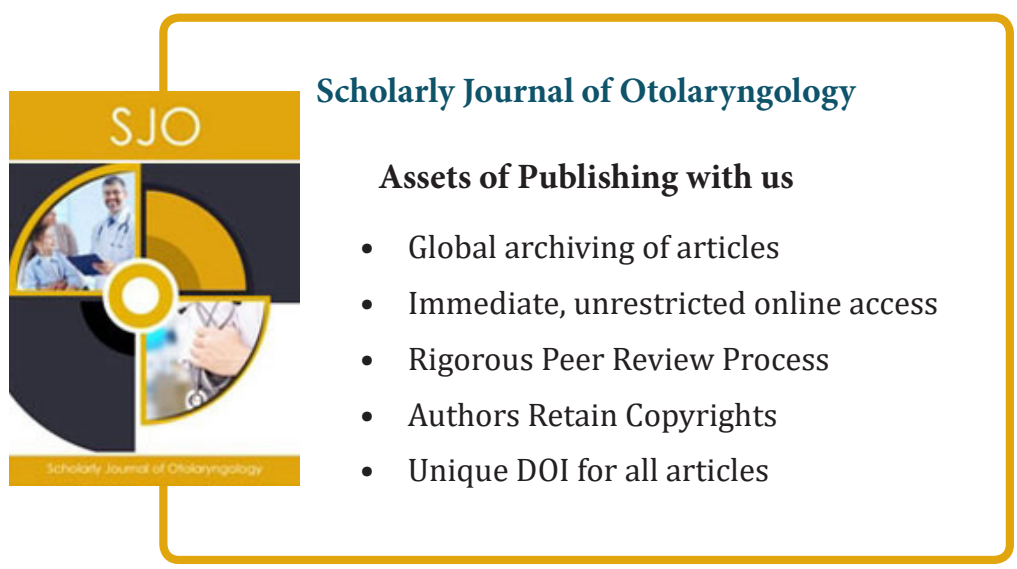

